\title{
Vestibular-Somatosensory Convergence in Head Movement Control During Locomotion after Long-Duration Space Flight
}

\author{
Ajitkumar Mulavara, ${ }^{1}$ Tara Ruttley, ${ }^{2}$ Helen Cohen, ${ }^{3}$ Brian Peters, ${ }^{4}$ Chris Miller, ${ }^{4}$ Rachel Brady, ${ }^{4}$ Lauren Merkle, ${ }^{4}$ \\ and Jacob Bloomberg ${ }^{2}$ \\ ${ }^{1}$ Universities Space Research Association, Houston, TX, USA, ${ }^{2}$ NASA Johnson Space Center, Houston, TX, USA, \\ ${ }^{3}$ Baylor College of Medicine, Houston, TX, USA, and ${ }^{4}$ Wyle Integrated Science and Engineering Group, Houston, \\ TX, USA
}

Exposure to the microgravity conditions of space flight induces adaptive modification in the control of vestibularmediated reflexive head movement during locomotion after space flight. Space flight causes astronauts to be exposed to somatosensory adaptation in both the vestibular and body load-sensing (BLS) systems. The goal of these studies was to examine the contributions of vestibular and BLS-mediated somatosensory influences on head movement control during locomotion after long-duration space flight. Subjects were asked to walk on a treadmill driven at $1.8 \mathrm{~m} / \mathrm{s}$ while performing a visual acuity task. Data were collected using the same testing protocol from three independent subject groups; 1) normal subjects before and after exposure to 30 minutes of $40 \%$ bodyweight unloaded treadmill walking, 2) bilateral labyrinthine deficient (LD) patients and 3) astronauts who performed the protocol before and after long duration space flight. Motion data from head and trunk segmental motion data were obtained to calculate the angular head pitch (HP) movements during walking trials while subjects performed the visual task, to estimate the contributions of vestibular reflexive mechanisms in HP movements. Results showed that exposure to unloaded locomotion caused a significant increase in HP movements, whereas in the LD patients the HP movements were significantly decreased. Astronaut subjects' results showed a heterogeneous response of both increases and decreases in the amplitude of HP movement. We infer that BLS-mediated somatosensory input centrally modulates vestibular input and can adaptively modify head-movement control during locomotion. Thus, space flight may cause a central adaptation mediated by the converging vestibular and body load-sensing somatosensory systems. 